Document downloaded from:

http://hdl.handle.net/10251/43816

This paper must be cited as:

Sendra Compte, S.; Granell Romero, E.; Lloret, J.; Rodrigues, JJPC. (2014). Smart Collaborative Mobile System for Taking Care of Disabled and Elderly People. Mobile Networks and Applications. 19(3):287-302. doi:10.1007/s11036-013-0445-z.

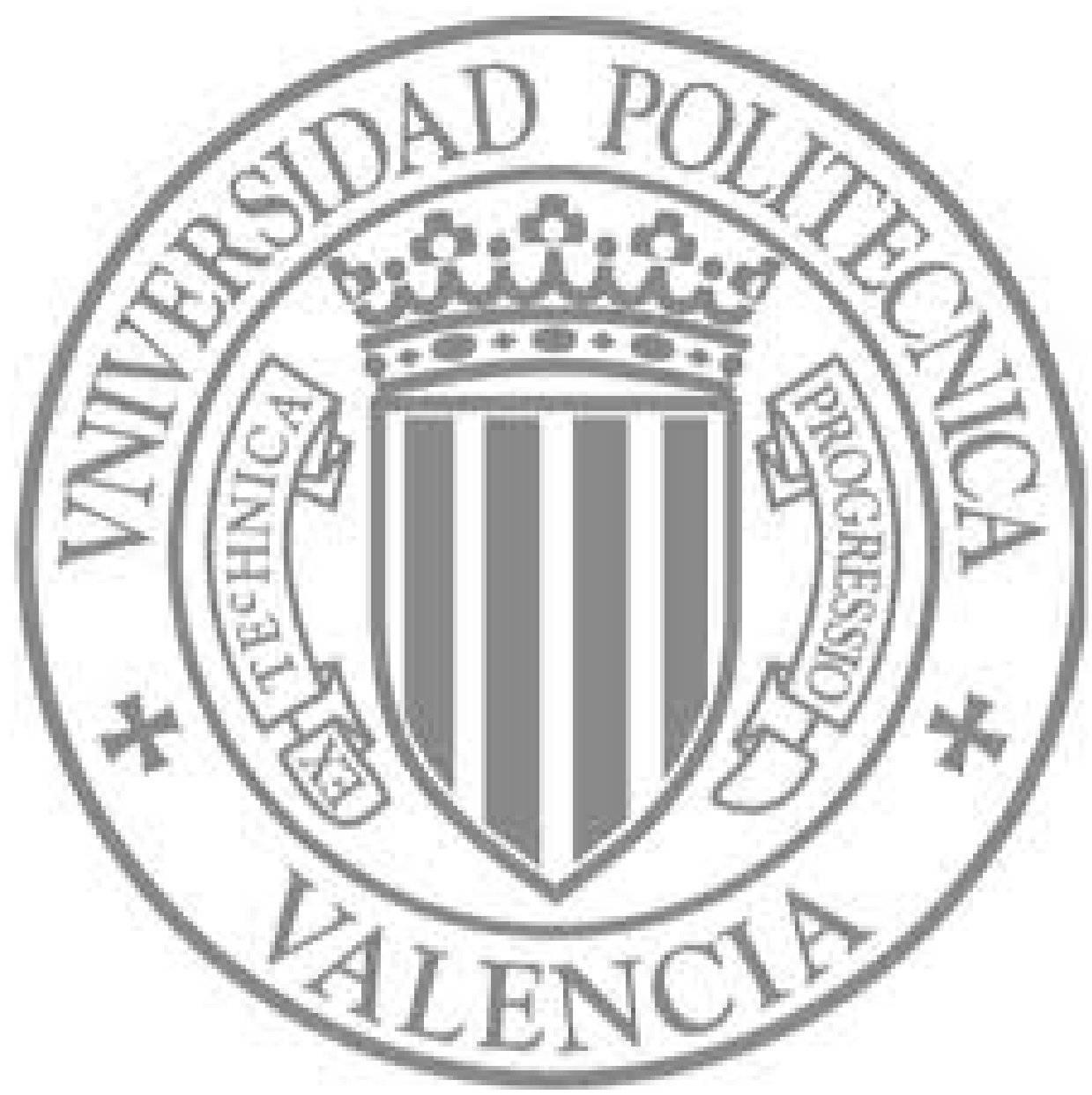

The final publication is available at

http://dx.doi.org/10.1007/s11036-013-0445-z

Copyright Springer Verlag (Germany) 


\title{
Smart Collaborative Mobile System for Taking Care of Disabled and Elderly People
}

\author{
Sandra Sendra ${ }^{\mathrm{a}}$, Emilio Granell ${ }^{\mathrm{a}}$, Jaime Lloret $^{\mathrm{a}}$, Joel J. P. C. Rodrigues ${ }^{\mathrm{b}}$ \\ ${ }^{a}$ Integrated Management Coastal Research Institute, Universidad Politécnica de Valencia, C/Paranimf, $n^{\circ} 1$, Grao de Gandia, 46730, Spain \\ ${ }^{b}$ Instituto de Telecomunicações, Universidade da Beira Interior, Rua Marquês d'Ávila e Bolama, Covilhã, 6201-001, Portugal
}

\begin{abstract}
Official statistics data show that in many countries the population is aging. In addition, there are several illnesses and disabilities that also affect a small sector of the population. In recent years, researchers and medical foundations are working in order to develop systems based on new technologies and enhance the quality of life of them. One of the cheapest ways is to take advantage of the features provided by the smartphones. Nowadays, the development of reduced size smartphones, but with high processing capacity, has increased dramatically. We can take profit of the sensors placed in smartphones in order to monitor disabled and elderly people. In this paper, we propose a smart collaborative system based on the sensors embedded in mobile devices, which permit us to monitor the status of a person based on what is happening in the environment, but comparing and taking decisions based on what is happening to its neighbors. The proposed protocol for the mobile ad hoc network and the smart system algorithm are described in detail. We provide some measurements showing the decisions taken for several common cases and we also show the performance of our proposal when there is a medium size group of disabled or elderly people. Our proposal can also be applied to take care of children in several situations.
\end{abstract}

Keywords: disabled and elderly people, mobile wireless network, collaborative system

\section{Introduction}

Several studies show that nearly one mobile device per capita is expected in 2015 [1]. The features of these smart devices have increased hugely and this fact has increased their popularity among people, especially between the young ones. Current mobile phones are being considered as the new portable computers that can also be used to process data and communicate with other smart devices. In addition, recent studies show that smartphones are being used to capture data from sensors and display them to users [2]. The major advantages of these devices are that most of them are easy to wear, highly programmable and, in addition, they have a set of cheap and powerful embedded sensors, which can be used to measure the environment in order to achieve important goals.

In addition, last generation devices present high processing capabilities, large memory and several communication choices, such as, GSM, GPRS, UMTS, Wi-Fi, Bluetooth, Infrared, NFC, and GPS positioning technology.

One of the most current representative smartphones is the new Galaxy Nexus [3]. It has a $1.2 \mathrm{GHz}$ dual core processor with $32 \mathrm{~GB}$ of memory for storage and $1 \mathrm{~GB}$ of memory RAM. In addition, it has embedded an important set of sensors: accelerometer, magnetic sensor, gyroscope, compass, GPS, proximity/light, barometer, 2 cameras and 2 microphones. Galaxy Nexus permits to establish connections through GSM, EDGE, GPRS, UMTS, HSDPA, LTE/CDMA, WiFi, Bluetooth, NFC and Micro USB.

Disabled and elderly people are a population sector that is quite dependent. This dependency has several levels that depend on the grade of disability. In some cases they may require an assistant or supervisor to perform most 
of everyday tasks or even always need another person with them. But some others can do their daily lives and they only need an advising system because the probability of having a problem is higher than regular people.

According to the "Instituto Nacional de Estadística", in Spain, there are currently 3.847.900 people with some kind of disability in this country. Moreover, the population of the European Union (EU-27) is also aging rapidly. Several estimations suggest that the population group aged over 65 will increase from $17.4 \%$ in 2010 to $29.9 \%$ in 2060 [4]. This means that the percentage of disabled and elderly people is increasing.

Moreover, there are several studies, such as the one presented by K.E. Thomas [5], that show the importance of falls in the elderly people. They fall more often and they need to recover more time. Although unintentional falls are inevitable, it is important to do an early treatment because even a short falls could trigger a series of major problems, even death especially in the case of brain damage.

Current information and communication technologies can increase the disabled and elderly people independence and their quality of life, thus making possible their professional and cultural inclusion. Although many researchers are focused on deploying new Wireless Body Sensor Networks (WBAN) [6] with new efficient applications [7], many smartphone applications that use the embedded sensors are appearing [8]. So, it makes sense to take profit of all their benefits.

In this paper, we describe a smart system based on the embedded sensors of mobile devices in order to determine if a person that wears one of these devices has suffered an incident or has a problem, when the group of persons are doing an activity. It is an extension of our previous work [9]. In this new paper we have added the concept of reinforcement learning that allows our system to improve its accuracy to predict an alarm before it happens and also reduce the amount of false positives. Moreover we have increased the number of devices participating in the collaborative decision. We provide some simulations that show the performance of the proposed system when there is a medium size group of disable or elderly people. Different routing protocols perform different for different mobile conditions, connections and disconnections. Because of this, we compare four of the most used ad hoc network protocols when running our system conditions. Our purpose is to show which one is better for our system and how all of them perform in a particular case.

The rest of the paper is structured as follows. Section 2 shows some works related with the use of smart devices to monitor and control some environment parameters. The system implementation, proposed system operation and algorithm procedure are explained in Section 3. Section 4 shows several performance tests. It shows the system performance results when there are several alarms, when some alarms are mixed and its performance when using several routing protocols. Finally, section 5 draws the conclusion and future works.

\section{Related work}

This section reviews the related literature that takes profit of the smartphones' embedded sensors and shows some real life applications. We have also added other works that describe useful implementations to improve the disabled and elderly people quality of life.

Because millions of people carry mobile phones nowadays, many developers have unconsciously deployed a strong infrastructure which can help us to implement new proposals, based on the sensing capabilities of these devices. Following this idea, Nicholas D. Lane et al. [10], discuss the state of the art of mobile phone sensing. This work analyzes some aspects in many different disciplines such as business, healthcare, social networks, environmental monitoring, and transportation. In addition, they review the main sensors embedded in mobile phones and discusses which possibilities could open this technology to the society.

Because the processing capabilities of mobile devices are growing more and more, the incorporation of several sensors and increase the complexity of the applications needed to control all these sensors is possible. In this line, C. Muldoon et al. [11] propose the use of a Multi-Agent System called "Collaborative agent tuning" to optimize the system operation in devices with limited computing capacities. This solution permits developers to manage and control all available sensors in mobiles devices and smartphones. 
When we talk about wireless sensor networks, we think on networks formed by geographically dispersed sensors that work together to monitor the environment. The increased use of smartphones suggests developing large sensor networks using these mobile devices as sensor nodes [12]. In [13], Kansal et al. present a first attempt towards the understanding and implementation of a programmable sensor network using the infrastructure created by the different mobile devices. The proposed system uses the collected samples of the phone sensors and shares the data with other users publishing it in a data repository.

Demographic forecasts indicate that elderly population will increase exponentially in the future in developed countries, mainly because of the new advances in medicine and prevention illness systems. This trend makes the development and implementation of new supporting technologies imperative in the daily environment. In [14], Immaculada Plaza et al. outline the opportunities that mobile applications offer to improve the quality of life of the elderly people. An example of this idea is the TeleCARE project, presented by L. M. Camarinha-Matos and H. Afsarmanesh [15]. The main goal of this project is to develop a system for tele-supervision following a multiagent approach, which will facilitate independent lifestyles for elderly people. The infrastructure includes both fixed terminals and mobile phones and allows giving independence and better quality of life to elderly people and their families.

The advances of wireless network technology and the development of body sensors are helping to improve the care of patients with reduced mobility at hospitals and health centers [16]. Accidental falls in the elderly and disabled people is considered one of the major health problems, in terms of primary care costs, of the public and private health systems [5]. We found several approaches that use smartphones for elderly people falls detection. In [17], authors detect falls using the sensors of an Android smartphone. P. Martín et al. present in [18] a multiagent system for mobile devices that can detect the falls of people using the sensors embedded in the device. If a fall is detected, the system generates a message with the GPS position coordinates that is sent via SMS and at the same time the device can also make emergency calls. The presented proposal has low computational cost, which implies less power battery consumption. In addition, the traffic generated into the network is acceptable. It has a fast mechanism to reduce the impact of the falls for elderly people, while permits an automatic and quick response to the potential incidents.

Fall detection must be lightweight to mobile phone resources consumption. To achieve this goal we can use learning techniques like the semi-supervised algorithm used by Ali Fahmi et al. in [19]. Each user moves differently, for this reason, the system should have adjusted the classification parameters to the particular movements of the user. In [20], the authors propose an adaptive algorithm that allows the personalization of the patient profile.

The care of elderly people that live alone and the extension of their independence are the goals of the activities tracking system described by Muhammad Fahim et al. in [21]. Moreover, Boštjan Kaluža et al. presented in [22] a system with the same goals by using a multi-agent approach. It is able to send an alert when there is an emergency or unusual behavior.

A. Costa et al. [23] presented a system that allows health professionals knowing the vital parameters of the patients in their care. This system is developed for PDA or smartphones, and it is able to send information about a monitored patient which is provided by a set of sensors. It permits a remote diagnosis, avoiding critical situations in unsupervised environments which may lead to the death of the patient.

In [24], Reza Olfati-Saber et al., analyze the consensus and cooperation algorithms in networked multi-agent systems. This work presents several algorithms that provide rapid agreement between all mobile devices allowing effective task performance by self-organizing networked systems.

Finally, A. Arcelus et al. provided an overview of the smart home technology in [25]. Authors describe how a smart system could be implemented in a house in order to help elderly and disabled people. This system is similar to the one presented in our paper. But, on one hand, it can only be used indoors, and, in the other hand, the authors only provide some ideas and they do not show in detail the operation of their system. 
So, as far as we know there is not any smart collaborative system like the one presented in this paper which uses the information of the embedded sensors and takes decisions after knowing the information of the embedded sensors in its neighbors devices.

\section{System proposal}

The amount of sensors that a mobile device can integrate is quite high. Most of the smartphones available in the market include many of the sensors presented in this section. Although several sensors have not yet been integrated into mobile devices, we can see that the technology is already developed. Therefore, it is only a matter of time to have them embedded into the smartphones. In this section, we show the embedded sensors which could be used in our system. We also present the decision algorithm procedure included in our system.

Our purpose is to use our system in a group of disabled or elderly people when they are moving in a rural area or in a city, but it can be used indoors or in a fixed place. Moreover, the systemcan also be used to take care of children. Therefore the values of all sensors are similar (considering a tolerance). Given this fact, we can use the sensor values of our neighbor devices to compare whether the change of our sensor values are different of our neighbor sensors or not. Although it can be used in any type of environment, for design and development we will suppose the worst case: people are moving in an unknown place.

In our system each person has a smartphone that integrates several sensors and transducers, such as proximity/light sensor, magnetic sensor, acoustic sensor or microphone, compass, gyroscope, accelerometer, GPS locator or others, such as a barometer (see Figure 1). Our sensors do not measure physical conditions of the body. Devices only measure if a person has fallen, if he/she has changed his/her position, if there is more sun light, etc.

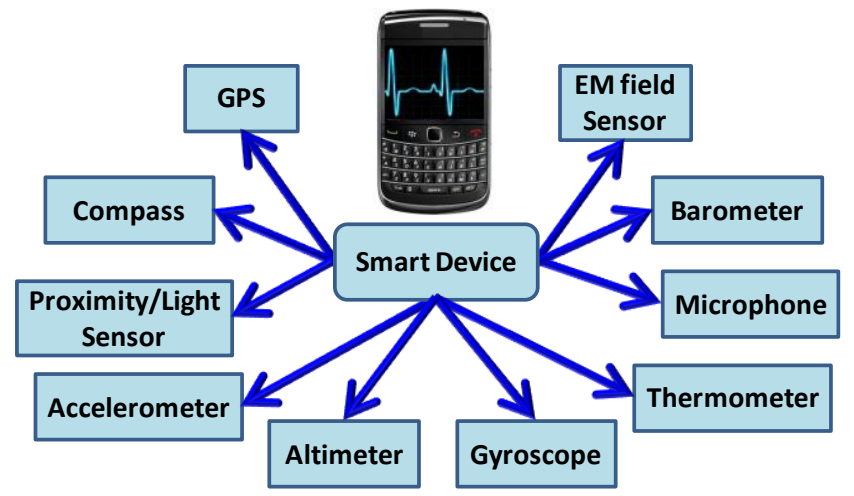

Figure 1. Sensors embeded in a smart device.

The most common embedded sensors in current smartphones are the following ones:

- Compass: An electronic compass is a navigational instrument that bases its operation in the terrestrial electromagnetic field. The magnetic needle indicates the direction of Earth's magnetic field, pointing to the north and south poles. With this sensor, we can determine the direction deviation from the North position in degrees. This sensor let us know in which direction group is walking. If a person is walking to another direction, the sensor will inform us.

- Accelerometer: This sensor is capable of measuring the acceleration and speed changes of an object. We can find several types of accelerometers. The first type is the Hall Effect accelerometers where a seismic mass with a magnet interacts with a Hall Effect sensor that detects the changes of the magnetic field. The second type is based on capacitors. In this case, seismic mass is placed between the plates of a capacitor. It changes the current between the capacitor plates when the mass moves. The third type of accelerometer is the three- 
axis accelerometer (X, Y, Z). It is integrated in a single chip of silicon. The principle of operation of these accelerometers is the MEMS (Micro Electric-Mechanical System) technology which is based on heat transfer by natural convection. These devices measure internal changes in heat transfer caused by acceleration, offering significant advantages over the use of a traditional structure based on solid proof masses. With a simple application it is easy to determine the distance traveled by the person. With the correct processing on the three axis signals, we can determine if a person is running or is going up on a lift. Moreover, the sensor can also inform us if the person has fallen. The average speed of a walking person is set to approximately $1 \mathrm{~m} / \mathrm{s}$.

- Gyroscope: It is a mechanical device consisting of a rotationally symmetric body which rotates around its axis of symmetry. When the gyroscope is subjected to a momentum, which tends to change the orientation of its rotation axis. Its behavior is apparently paradoxical, because the axis of rotation, rather than change the direction as a none-rotating body would do; it changes its direction perpendicular to the "intuitive" one. We can find several types of gyroscopes. The first type is the piezoelectric gyroscope. This is based on the use of an oscillating crystal. This oscillation arises when the acceleration acts on it and the crystal is deformed. Therefore its electrical resistance varies. The second type is the capacitive gyroscope, which is composed of two parts: a mobile part that performs an input/output vibrating movement regarding to the axis of rotation of the gyroscope and a fixed part, which is perpendicular to this movement. These two parts form a capacitive structure that can store an electric charge (as a capacitor). Finally, the third type is the MEMS gyroscope, which is based on the vibration but using materials at molecular level. This technology allows its integration in a small silicon chip and even mobile three-dimensional parts. The system analyzes the variations (which should be stable). The Coriolis Effect is used to calculate the variables. We can use gyroscopes to measure deviations from the reference direction. We can use a gyroscope to know if a person has fallen and he/she cannot get up. We will express the spin as a proportional value, between 0 (for the position repose) and 0.99 for a full circle.

- GPS: It is a satellite global navigation system that determines worldwide the position of an object, person or vehicle with a few meters of accuracy. We can even get few centimeters of accuracy by using differential GPS. The receiver must locate at least three satellites to determine its position. It receives signals identifying and indicating the clock time of each satellite. Based on these signals, the device synchronizes the GPS clock and calculates the time that takes the signals to get to the device. With this information it determines by triangulation the distance of each satellite to the measuring point. Knowing the distance, the relative position to the three satellites is easily determined, and then the absolute position or actual coordinates of the measuring point is easy determined. We will know de position for all devices using the GPS values with an accuracy of about $2.5 \mathrm{~m}$. Furthermore; we can calculate the speed of the person with the received data. Using the GPS, we can determine the position of each person in the group and if one of them leaves the group.

- Microphone: The microphone is an electroacoustic transducer. Its function is to translate the vibrations, due to the acoustic pressure exerted to the capsule by the sound waves, into electrical energy. It allows the registration, storage, processing and transmission of audio signals. We can find different types of microphones made of carbon, piezoelectric, dynamic, and capacitive, among many others. According to some studies provided by the WHO (World Health Organization), the sound levels, in sleeps hours should be $30 \mathrm{~dB}$, and cannot exceed $45 \mathrm{~dB}$, while, the ambient noise in a city is from $65 \mathrm{~dB}$ to $70 \mathrm{~dB}$. The levels for a man and a woman crying are $85 \mathrm{~dB}$ and $80 \mathrm{~dB}$ respectively. If a person is exposed to sound levels higher than $75 \mathrm{~dB}$, it can suffer several illnesses such as hypertension and heart problems.

- Proximity/Light sensor: A photoelectric sensor responds to the light intensity changes. There are several skin diseases, such as Lupus or photophobia, where patients cannot receive sunlight directly. In order to protect the people who suffer these diseases, we need to know the maximum light intensity for a person. 1200 lux is a low light intensity, while when there are 2800 lux, high light intensity (light outdoors) is considered. With this sensor we can detect the index of solar radiation that a person may be receiving. 
- Magnetometer: It is used to detect the magnetic field strength variation. Since the magnetic field influence decays with the distance., it is important to place the magnetometer as closer as possible to the target. It can be used as a metal detector.

- Temperature sensor: A temperature sensor can be manufactured with many configurations, differing in their optical and electronic components, its technology, size and package. For the implementation of temperature sensors in mobile devices such as PDAs or smartphones, it is commonly used resistive temperature sensors or semiconductor sensors, whose operation is identical to a diode. The temperature is given as an indirect measure of the variation of electrical parameters of these electronic components.

- Altimeter/Barometer sensor: Its operation is based on the relationship between atmospheric pressure and altitude above sea level [26]. Atmospheric pressure decreases with altitude, about $1 \mathrm{hPa}$ per 27 feet $(8.2$ meters) above the sea level. It is taken as baseline the sea level, but its operation is subject to weather changes. For this reason, a single device usually gives both values.

Although the smartphone used for this work does not integrate the temperature and altimeter sensors, other mobile phone models, made for sports people, may integrate them [27]. Furthermore, it has been recently developed a precision analog output temperature sensor for low current applications (STLM20 manufactured by STMicrorelectronics) [28]. These sensors will most probably be integrated into new smartphones soon.

\subsection{Smart decision algorithm}

Initially, we define a threshold for each sensor. It will be used by the algorithm to decide whether there is an emergency case or not. Each sensor has different precision, so we must take into account their errors. Table 1 shows the errors and the thresholds taken into account for previous sensors. Using these values we can see which measurements are tolerable and when alarms should be sent.

The problem key in a system of monitoring elderly and disabled is to detect situations in which a user needs help. In our system we have formulated the policy of alarm as a Markov Decision Process (MDP) and reinforcement learning.

\begin{tabular}{l|ll}
\hline Sensor & Error & Threshold \\
\hline Compass & 1 degrees & 10 degrees \\
Accelerometer & $1 \mathrm{~cm}$. & $10 \%$ \\
Gyroscope & $1 \%$ & $5 \%$ \\
GPS & $2.5 \mathrm{~m}$ & - \\
Microphone & $1 \mathrm{~dB}$ & $10 \%$ \\
Proximity/Light sensor & $0.02 \mathrm{lux}$ & $2500 \mathrm{lux}$ \\
Magnetometer & $1 \mu \mathrm{T}$ & $10 \%$ \\
Temperature sensor & $1^{\circ} \mathrm{C}$ & $10 \%$ \\
Altimeter/Barometer & $1 \mathrm{~m}$ & $20 \mathrm{~m}$ \\
sensor & & \\
\hline
\end{tabular}

Table 1. Errors and thresholds of the sensors.

Formally an MDP is a tuple $\langle S, A, P, R\rangle$, where:

- The finite set of states $S=\left\{s_{1}, \ldots, s_{n}\right\}$ is a set of situations in which a user can be found by its neighbors. Each state is represented by a feature vector, where each component represents whether the magnitude of the difference between the sensor value of the phone and the mean values of the neighboring sensors exceeds a threshold, so if it is equal to or greater than this threshold will take a value of 1 and 0 if less.

- The set of actions $A=\left\{a_{1}, \ldots, a_{k}\right\}$ is a finite set of actions. In our system the possible actions are "Wait" and "Send an alarm message". 
- $P: S \times A \rightarrow S^{\prime}$ is a state transition model, which determines the probability of reaching a state $s^{\prime}$ from a state $s$ by executing action $a$. Since we are in an unknown stochastic environment, the transition probabilities between states are unknown. For this reason, we apply the technique of Q-Learning reinforcement learning.

- $R: S \times A \times S^{\prime} \rightarrow R$ is the expected reward function, which determines what reward or penalty will receive the system if choosing the action $a$ from state $s$ reach the state $s^{\prime}$. The reward is the accuracy of detection of dangerous situations, so false positives are penalized with -1 while the true positives are rewarded with +1 .

The utility value of each action in each state $\mathrm{Q}(s, a)$, at the start of learning, is defined in a table according to our criteria of alarms which will be explained later, during the use of the system the utility values are updated by Equation 1.

$$
Q_{t}(s, a) \leftarrow Q_{t-1}(s, a)+\alpha\left[R_{t}(s, a)+\gamma \cdot \max \left(Q_{t}\left(s^{\prime}, a^{\prime}\right), Q_{t-1}(s, a)\right)\right]
$$

Where $Q_{t}(s, a)$ is the new utility value, $Q_{t-1}(s, a)$ is the above utility value, $\alpha$ is the learning factor, $\gamma$ is the discount factor for future values, $R_{t}(s, a)$ is the immediate reward and $\max _{a^{\prime} \in A^{\prime}} Q\left(s^{\prime}, a^{\prime}\right)$ is the utility value of the optimal action chosen in the future state.

Q-Learning reinforcement learning techniques allow our system to predict an alarm before it happens and also reduces the amount of false positives, thus improves the system accuracy.

We have chosen a deterministic policy. So for a given state it will choose the action that maximizes the utility value. It is represented by equation 2 .

$$
a=\arg \max _{a \in A} Q(s, a)
$$

The algorithms deployed for the proper running of the proposed system are shown in Algorithms 1, 2 and 3 . When the system starts, it runs Algorithm 1. This algorithm constantly calls Algorithm 2 for monitoring the current status of the device and the action to execute. The chosen action is executed and then Algorithm 1 calls Algorithm 3 in order to obtain the reward for executing that action. In this point, the user can act on the system in order to determine if it was a false positive, so the system learns from it for the next time. To complete the cycle, the system updates the utility value of the action performed in the previous state.

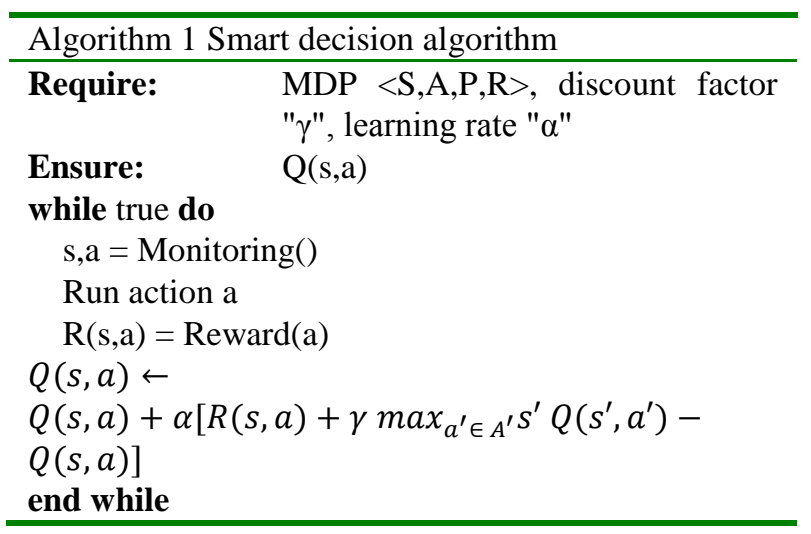

\begin{tabular}{lc}
\hline Algorithm 2 Monitoring \\
\hline Input: & List of own sensors list \\
Output: & State s, action a \\
list & $\leftarrow$ getNeigborgs () \\
for all sensor $\mathrm{S}_{\mathrm{j}}$ in list & do \\
\hline
\end{tabular}




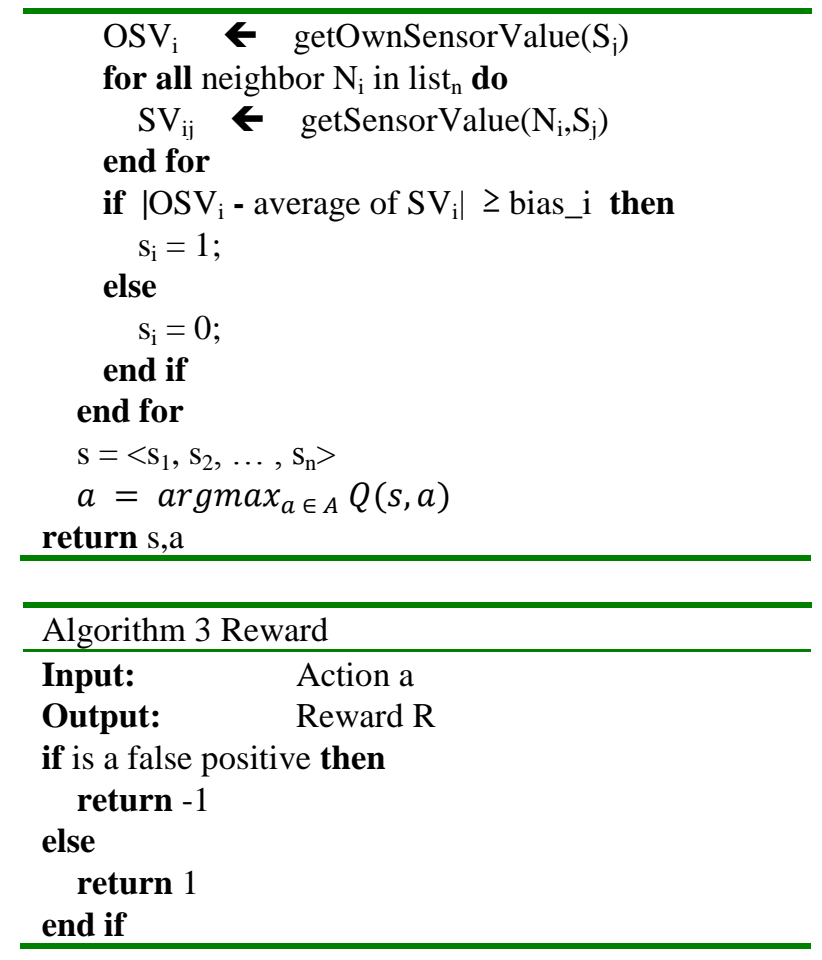

\subsection{System operation}

With a correct signal processing of the sensor values of the state $s$ and comparing these values with the values of its neighbors, our system is able to detect some situations or events that are beyond ordinary cases, such as:

- A person that is falling.

- The distance between a person and the group is increasing.

- A person is screaming, but it is not the noise of the place.

- A person with a skin disease is receiving sunlight.

- A person is moving in a different direction to the group direction. Moreover, this system would allow us to distinguish other types of events that could bring the system to generate alarm signals when in certainty the situation does not require it:

- A device detects high noise, so a regular system will generate an alarm thinking that the user is crying or shouting, but he/she is very close to the neighbors to which the system will ask for the value of their sensors. Because levels picked up by its neighbors are very similar, the alarm would not be sent.

- A person is walking backwards but with the group. It generates the alarm by the compass sensor because it detects a change of direction. Without a collaborative system this issue will not be advised.

- A person scampers near the group. It would generate the alarm because of the difference of the values of the sensors. However, it may not be an alarm because the person is not moving away from the group.

- One person performs reciprocating movements. When the system compares the value of the sensors with the values of the nearest neighbors, it would generate the alarm because of their differences. But this situation does not involve an emergency case.

There could be many more cases. An alarm is generated as a combination of the value sensors of the device, but after considering the received replies from the neighbors. Therefore, our system considers the following statements:

- Difference of sensor values with respect to the previous values. 
- Difference of the current state with the sensor values of the neighbors.

- The combination of several alarms, to distinguish between a true emergency situation and a situation that has generated a false positive.

As Figure 2 shows, we consider a group of people traveling together (or in a same place). We are thinking on people that must be always monitored, which can have Alzheimer or other issues such as degenerative diseases. The system is also suitable for monitoring children. In either case, each person is carrying a smart device connected to a wireless ad hoc network using IEEE $802.11 \mathrm{~b} / \mathrm{g}$ technology, where each device maintains wireless connections with their nearest neighbors.

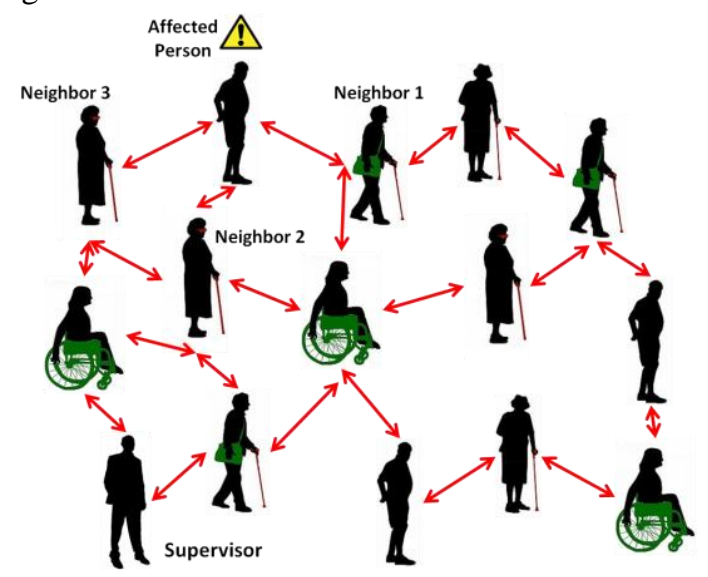

Figure 2. Network Topology.

Our proposal works with the same power saving approaches and energy optimization techniques as regular systems [29]. Our system will generate more messages when a threshold is exceeded. Only in this situation, there will be more exchanges of messages. So, we take profit of existing systems.

In order to determine whether an alarm must be announced or not, we have split the alarm procedure in four stages. Figure 3 shows the sequence of alarms as a function of the time. The procedure is as follows. The device is always monitoring the values from the sensors by using a small application that collects the value of each sensor. When the smartphone detects that a sensor has exceeded its threshold the system goes to the first alarm level. If after some time it is not disabled, it goes to the second level, where it sends a message to its closest neighbors requesting their values for this sensor.

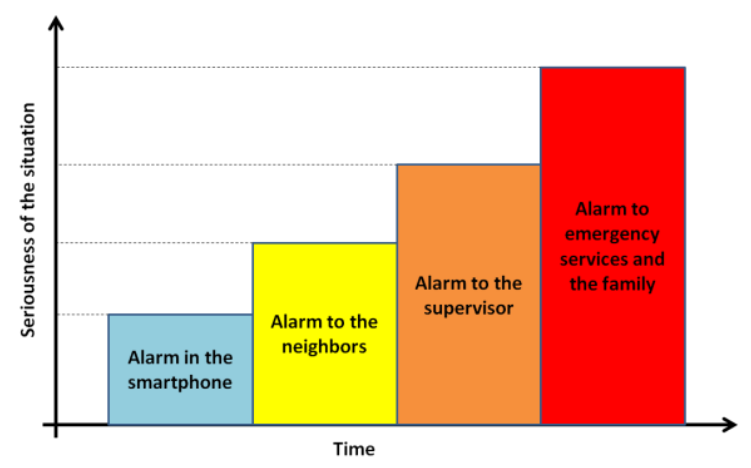

Figure 3. Alarm levels and propagation in function of the time. 
When the reply is received, it compares the received values with its values. If the result of the comparison of both values is very similar (considering the accuracy of each sensor), it will mean that the system has detected a false-positive, and the system will return to its resting state, monitoring the sensors activity. But, when the compared values are very different, it means that there is a real warning, generated for example, by the fall of a person, or because a person has been separated from the group. Then, it goes to the third level, where the device of the affected person sends a message with the cause of the alarm to the person in charge of the group (the guide or supervisor). If after some time the alarm is still active, because no one has stopped it, it will go to the fourth level. At this level, the smartphone will send an automatic message to emergency services and to the family. They will be able to determine the location of the person, thanks to the GPS coordinates.

When a device needs information from its neighbors, the first step is to know its neighbors. Initially, the affected person device sends a broadcast packet. Only nodes that are closer to him will reply. After assessing the situation (based on the sensor values), if the system decides to send an alarm, it will follow the four stages alarm procedure. When the alarm is sent to the supervisor, it is routed using a regular routing protocol (routing protocols are discussed in the performance test section). Supervisor will respond with an acknowledgment packet. Figure 4 shows the message flow between the affected person, neighboring nodes and the supervisor.

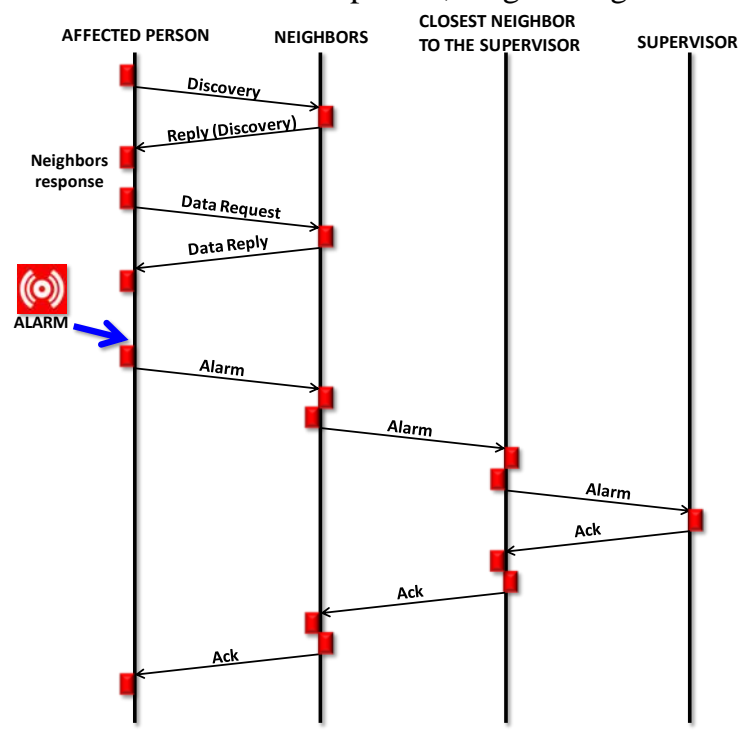

Figure 4. Message flow between devices

Figure 5 shows the collaborative decision algorithm which is in charge of processing the data from the sensors and determine if there is an alarm and whether the detected alarm is a real one or a false positive. Figure 6 shows the real code used in an Android mobile device in order to gather its sensor values. It shows how sensor values are taken from the device. 


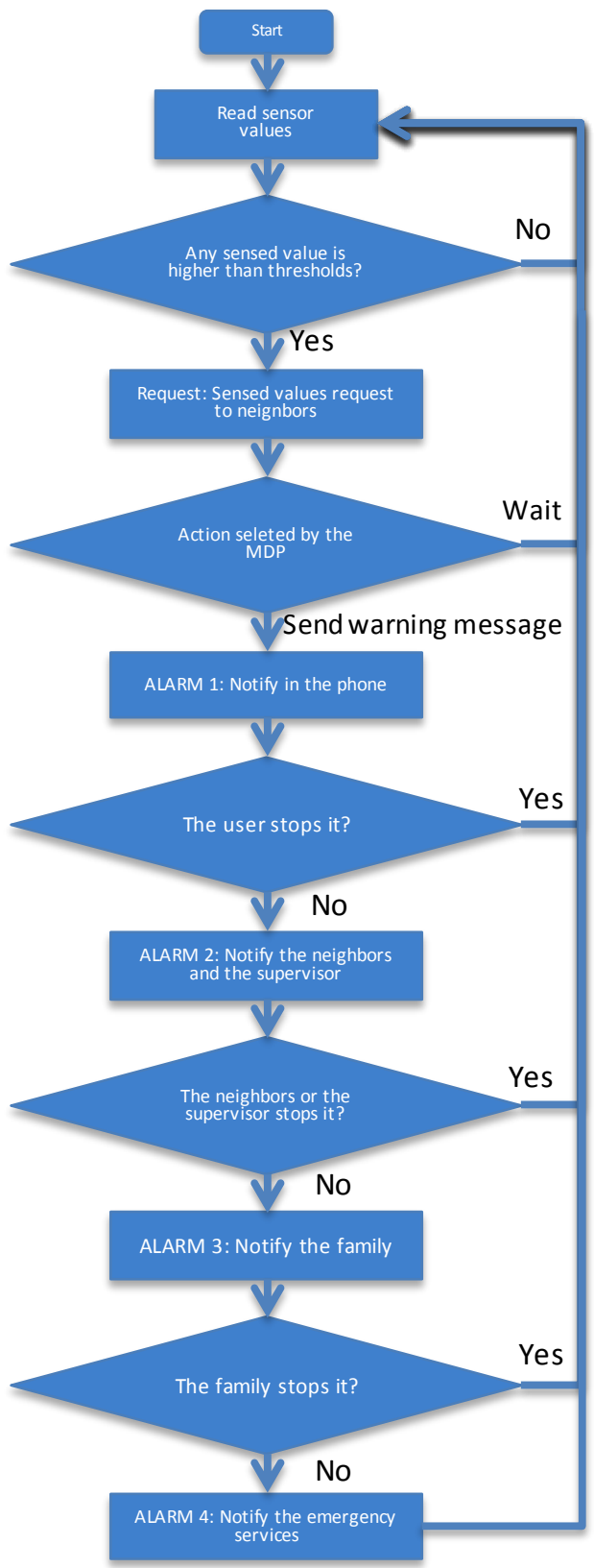

Figure 5. Collaborative decision algorithm.

public void onSensorChanged(SensorEvent event) \{

synchronized (this)

switch (event.sensor.getType()) \{

case Sensor.TYPE ORIENTATION:

for (int $\mathrm{i}=0 ; \mathrm{i}<3$; $\mathrm{i}++)\{$

orientation[i] = event.values[i]; 


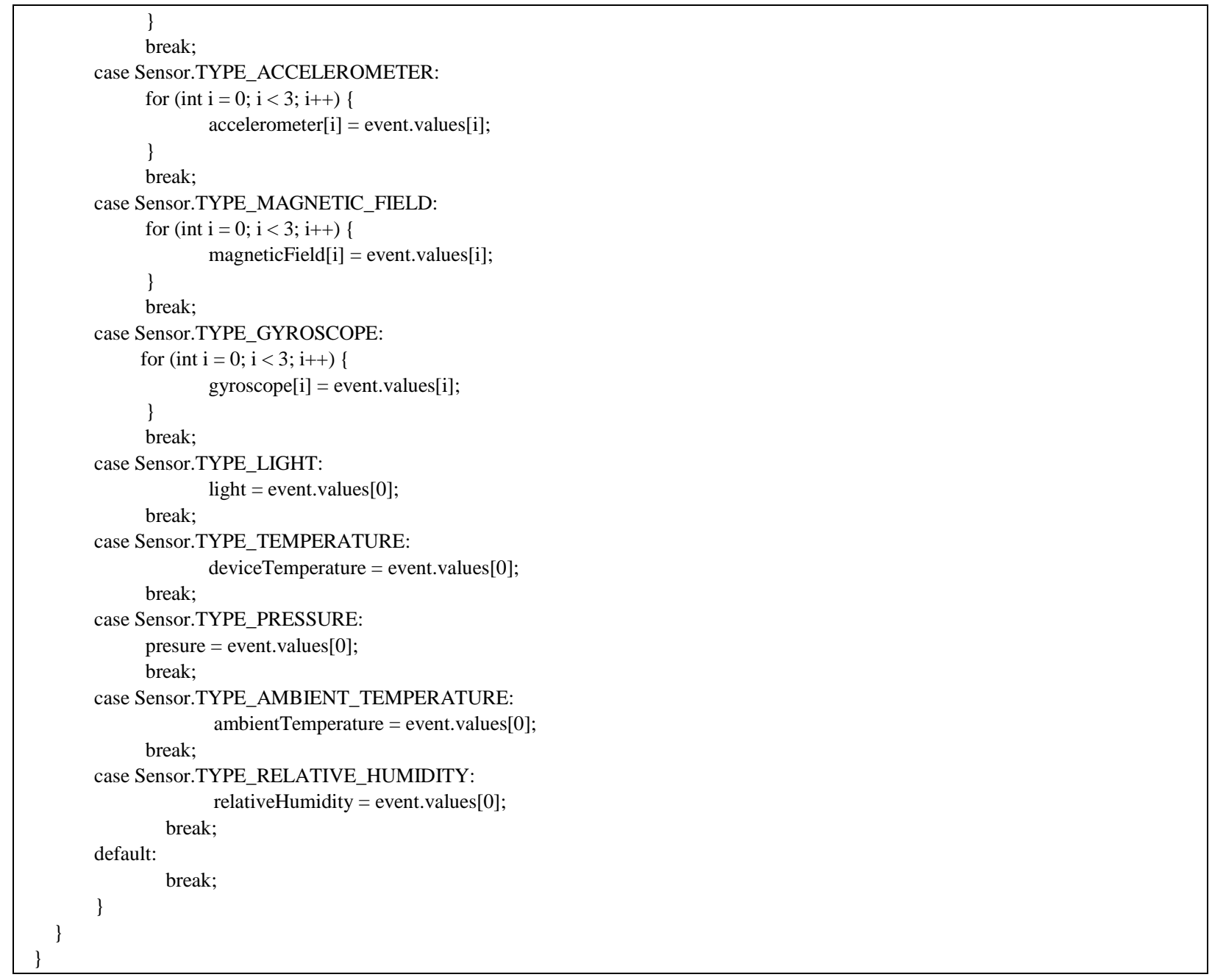

Figure 6. Function for reading the sensor values

\section{Performance test}

\subsection{System operation performance}

In this subsection we simulate a scenario like the one shown in figure 2. Initially, the mobile device of the affected person produces an event. Before creating a local alarm state, each device checks if its values exceed a threshold or if the parameters vary from their previous states. If one of these cases occur, the value that has generated the local alarm will be compared with its nearest neighboring nodes. Then, the affected device sends a request to its closest neighbors in order to compare its value with their values. If there is a difference higher than a threshold, the device generates a warning message to the supervisor. We have simulated our system using Matlab [30]. It is a high-level technical computing language and an interactive environment for algorithm development, data visualization and analysis. 
In the first case the system monitors a person that has changed its direction. The group direction changes can be tracked using the compass sensor. 100 samples of the compass sensor of 4 devices are collected. From the $27^{\text {th }}$ sample, one of the devices starts to change its travel direction (the affected person) compared to its neighbors (neighbor 1, neighbor 2 and neighbor 3), which follow the same path. Figure 7 shows this situation.

Moreover, in this case we can also include joint sensor values. E.g. the detection of a person that although he/she is walking with the group, he/she has moved around and walked backward, changing the direction of the person but going in the same direction of the group (he/she will not increase the distance to the group.

The second case monitors a person that is screaming for help. Figure 8 shows the sound level that is recorded on a device on a tour of an urban area, in $\mathrm{dB}$. From the $35^{\text {th }}$ sample, the device of the affected person has values greater than $80 \mathrm{~dB}$, indicating that this person is screaming. Neighbor 1, neighbor 2 and neighbor 3 present similar values, which may be registered in urban environments. As we have seen, in an urban environment, noise levels means are located around the $65-70 \mathrm{~dB}$. This is the background noise we have over our measurement.

There are specific cases where to measure a person crying could be difficult. When an ambulance passes with the siren on (depends on state law), it exceeds the average noise values in urban environment. It is often placed at $80 \mathrm{dBs}$. In this case, we would not be able to determine if the person is in a danger situation, so the query to other sensors should be necessary. There is another way to detect whether the increased noise level produced by a shout or an emergency vehicle. This would be possible if the system is able to distinguish the frequency of the received sound by using a simple frequency analysis.

In the third case, the system monitors a fixed place where it is expected to not have any leaving from that place. In the following scenario we have fixed a location to relate the devices. Figure 9 shows the distance that each monitored node presents to the fixed place (it could be the place of the supervisor). We can see that neighbor 1, neighbor 2 and neighbor 3 are always around $5 \mathrm{~m}$ away, while the affected person starts to leave the group, from sample $40^{\text {th }}$.

In the forth case the system monitors if a person leaves a building or a covered place and is exposed to the sunlight. There are persons that may be affected by sunlight exposure because they suffer some sort of photophobia or hypersensitivity. Figure 10 shows the light intensity in lux received by 4 persons in the same place. The intensity value remains close to 2000 lux in almost all samples, except from $80^{\text {th }}$ and $95^{\text {th }}$, where there is a light intensity greater than 3000 lux. In this case, the device sends a warning message only if the person wearing the device has that disease.

In order to show the behavior (using the gyroscope) when a person has fallen, or is moving strangely, we have included the fifth case. The gyroscope sensor included in the smartphone provides the variation produced in each axis. In this case, we monitor separately the values of the 3 axes in 4 devices. Figures 11, 12 and 13 show the gyroscope behavior in its $\mathrm{X}, \mathrm{Y}$ and $\mathrm{Z}$ axes respectively. We can see how the system performs when a person falls down. It usually does not affect only to one axis, in this case two axes are involved, but we can see some impact in the third axis. In the three figures, we can see that after sample $33^{\text {rd }}$ the values for the 3 neighbors remain similar, while the values for the person that has tripped are changing.

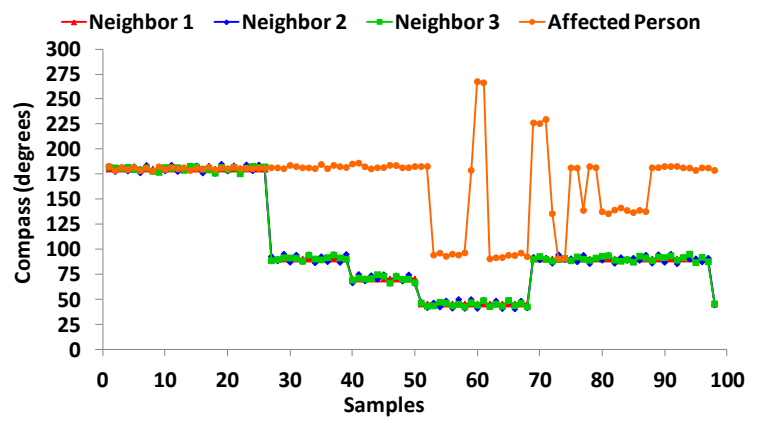

Figure 7.
Compass measurements for 4 devices

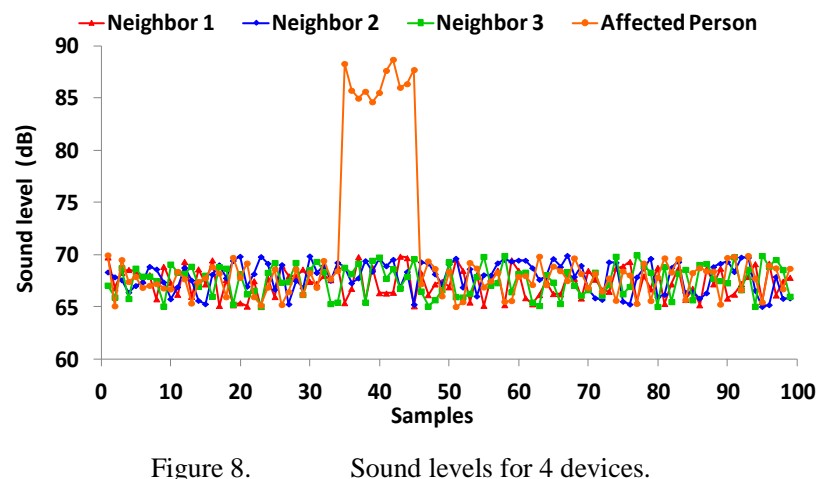

Figure 8. Sound levels for 4 devices. 


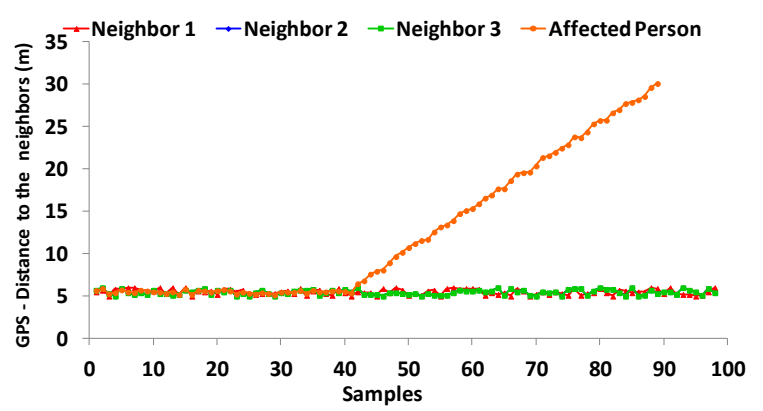

Figure 9. GPS measurements for 4 devices

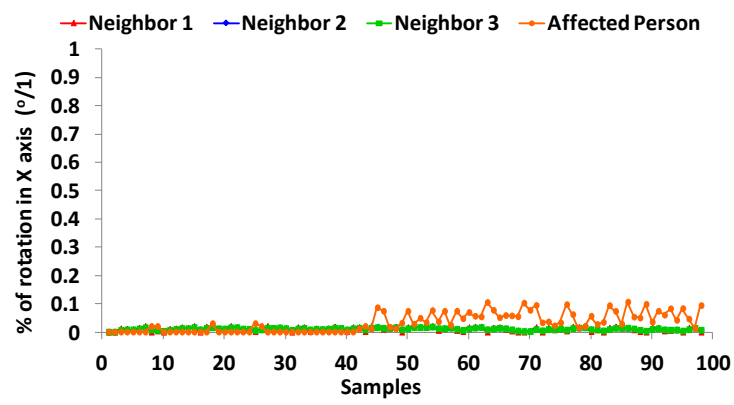

Figure 11. Gyroscope measurements in $\mathrm{X}$ axis for 4 devices.

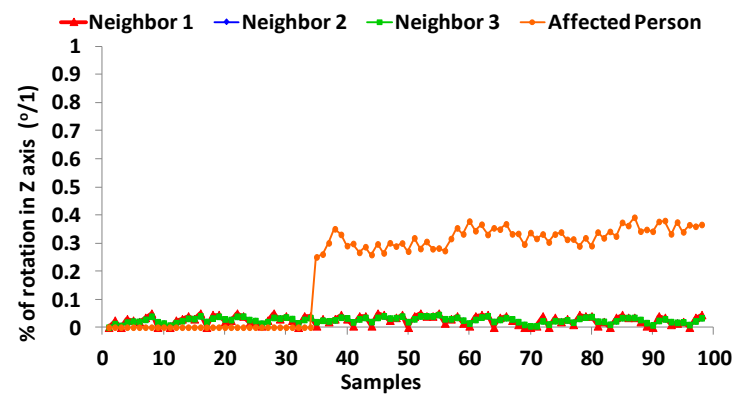

Figure 13. Gyroscope measurements in $\mathrm{Z}$ axis for 4 devices.

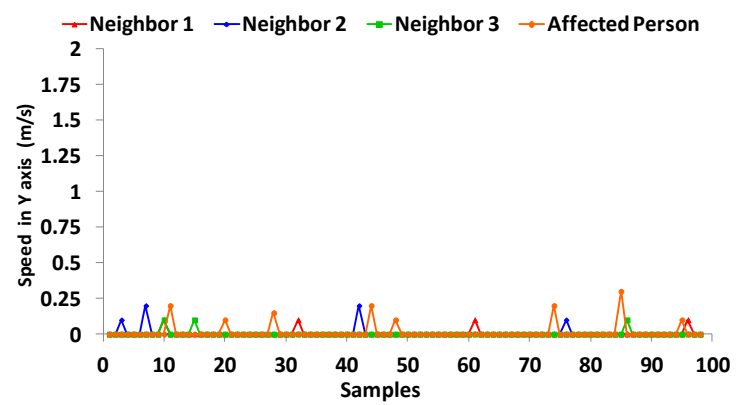

Figure 15. Accelerometer measurements in $\mathrm{Y}$ axis for 4 devices.

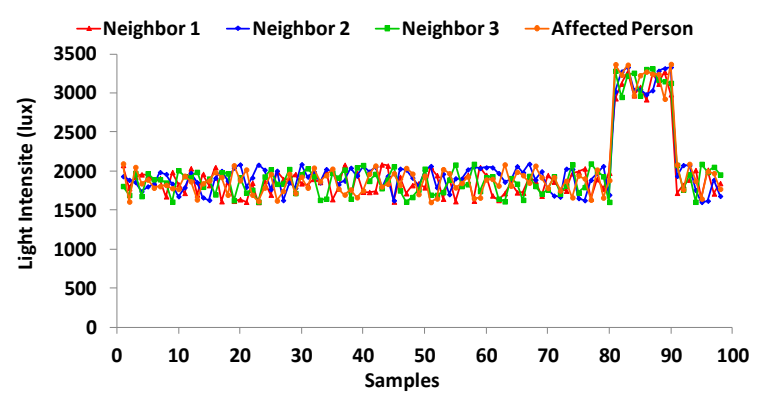

Figure 10. Light intensity measurements for 4 devices.

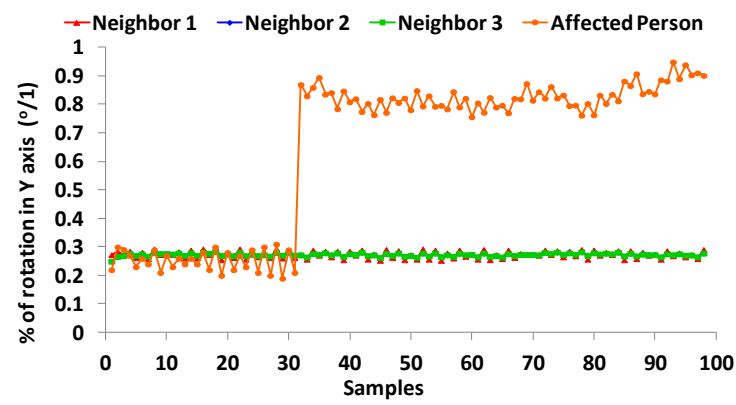

Figure 12. Gyroscope measurements in Y axis for 4 devices.

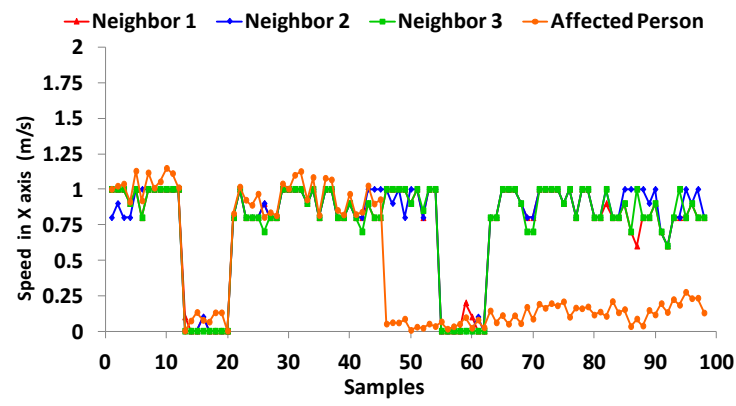

Figure 14. Accelerometer measurements in $\mathrm{X}$ axis for 4 devices.

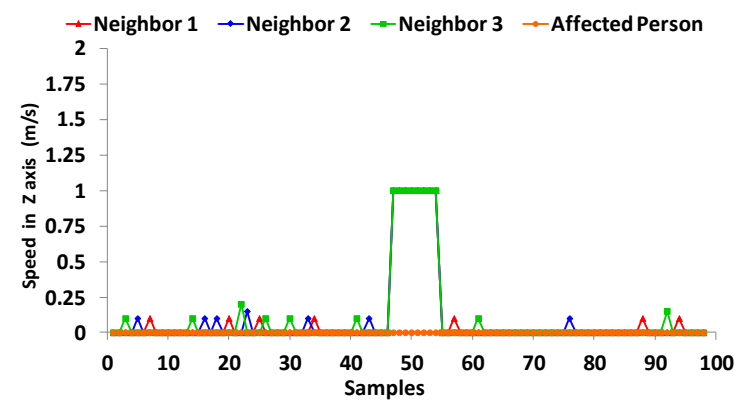

Figure 16. Accelerometer measurements in $\mathrm{Z}$ axis for 4 devices. 
In order to show the behavior (using the accelerometer) when a person has fallen or is moving strangely, we have included the sixth case. An accelerometer is capable of measuring the same behavior in the three axes than the previous case. We have also monitored separately these values for 4 devices. Figures 14, 15 and 16 show the accelerometer behavior in its $\mathrm{X}, \mathrm{Y}$ and $\mathrm{Z}$ axes respectively. We can see that the largest variations are recorded in the $\mathrm{X}$ axis, which is the direction of the movement of the group. First, we observe that between samples $13^{\text {th }}$ and $21^{\text {th }}$, all neighbors reduce their speed to $0 \mathrm{~m} / \mathrm{s}$, which means that they have stopped. Then, they continue walking. At sample $45^{\text {th }}$ we can see that something has happened to the affected person, because the smartphone detects different values in the $\mathrm{X}$ axis than its neighbors. This is because the person has tripped in its way. We can see that, at this sample, $\mathrm{Z}$ axis also detected a variation in its values. Because neighbors 1,2 and 3 notice the fall of the affected person, they also stopped between samples $55^{\text {th }}$ and $62^{\text {th }}$. Variations on the $\mathrm{Y}$ axis are very subtle, and may be due to small movements of the person in this axis. Finally, neighbors 1, 2 and 3 continue on their way and they increase their value in the $X$ axis, while the affected person maintains its value very close to $0 \mathrm{~m} / \mathrm{s}$.

\subsection{Alarms}

From the graphs shown in the previous section, and taking into account the measurements captured by the sensors, the device decides whether the affected person is in a dangerous situation and if it has to transmit the alarm message. This assessment is done by the MDP, depending on the state of the system defined by its sensor values compared with the sensor values of the neighbors, and the utility value of the alarm action for this state. In this section, we will see the generating of alarms starting from the cases seen in the previous section.

Figure 7 (first case) shows the alarm of the compass sensor. The four signals are analyzed, using the smart decision algorithm, in Figure 18. We can observe that as soon as the there is a variation between the affected person and its neighbors, the system starts to process the alarm. This occurs, starting from sample $27^{\text {th }}$. Moreover, we can see that in samples $73^{\text {rd }}-75^{\text {th }}$, the alarm is deactivated and then is reactivated. This means that although the affected person does not follow the same direction as the rest of the group during this short time period, the affected person has walked in the same direction than the group.

In the second case (Figure 8), we have processed signals from the microphones of 4 neighbors. Figure 17 shows the alarm generated in the device of the affected person. In it, we can see that from the sample $35^{\text {th }}$, a high level of alarm is established until it reaches sample $45^{\text {th }}$, where noise levels are restored and thus the alarm is disabled.

Another sensor that is very important for our system is the signal from the GPS sensor. We can use it to determine if the person is separated from the rest of the group (third case). Figure 19 shows the state of the alarm of the GPS sensors of the four neighbors. The alarm is activated from sample $46^{\text {th }}$.

The alarms generated by the light sensors work differently than the other sensors. The detection of high levels of light, above a threshold, might not be dangerous for a person, unless it suffers of some kind of photosensitivity (fourth case). Therefore, the algorithm is executed differently. In this case, the value of light collected by the sensor is firstly compared with the threshold value and then, it is compared with its neighbors, to check that the measured value is correct. If the comparison result is positive, the signal alarm is generated in the device of the affected person and it is propagated following the algorithm. Figure 20 shows the state of the light alarm. In this case, we see that the alarm is activated between samples $80^{\text {th }}$ and $91^{\text {st }}$.

The generation of the alarm for the gyroscope and accelerometer sensors (fifth and sixth case) is different from the rest. In these two cases, the alarms are calculated as a combination of the response of the $\mathrm{X}, \mathrm{Y}$ and $\mathrm{Z}$ axis, for each sensor. If we examine Figures 11, 12 and 13, corresponding to the behavior of the axes $\mathrm{X}, \mathrm{Y}$ and $\mathrm{Z}$ of the gyroscope sensor, we can see that the largest variations occur in the $\mathrm{Y}$ and $\mathrm{Z}$ axis. The alarm of the gyroscope sensor is obtained as a combination of the three signals. As Figure 21 shows, after the $31^{\text {st }}$ sample there is a series of activations/deactivations of the alarms, which could generate instability of the system. 
Following the same approach, the behavior of Figures 14, 15 and 16, have been combined. Figure 22 shows the alarm of the acceleration sensor. We see that between sample $58^{\text {th }}$ and $63^{\text {rd }}$, the alarm turn off. However, and for the same reasons as in the previous cases, it does not mean that the alarm situation has disappeared.

\subsection{Decision rules by mixing alarms}

In the previous section, we have seen several alarms that are activated depending on the data obtained by each sensor and their comparison with the values collected by its neighbors. However, if we treat the alarms for each sensor separately, we could get false positives. For this reason, we use the state alarm of the entire sensors to construct the state vector for the MDP. MDP decides when to send the alarm message combining the alarm signals from various sensors.

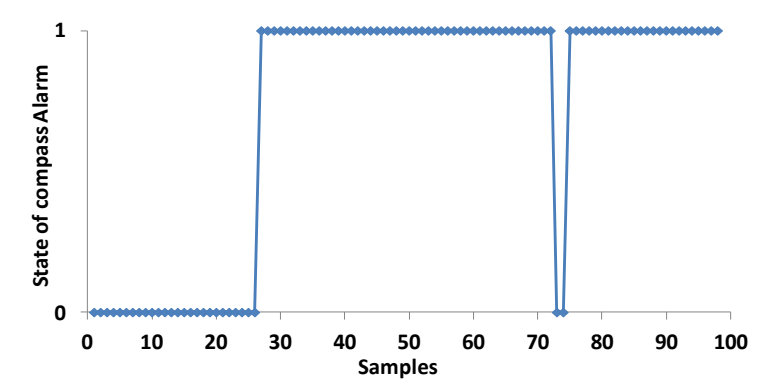

Figure 17. State of compass alarm

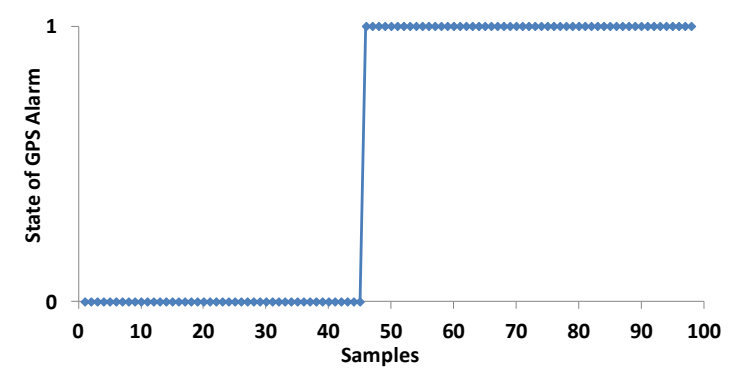

Figure 19. State of GPS alarm

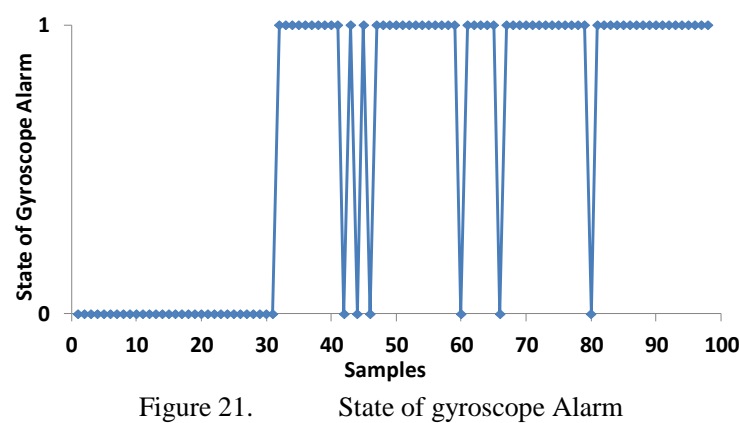

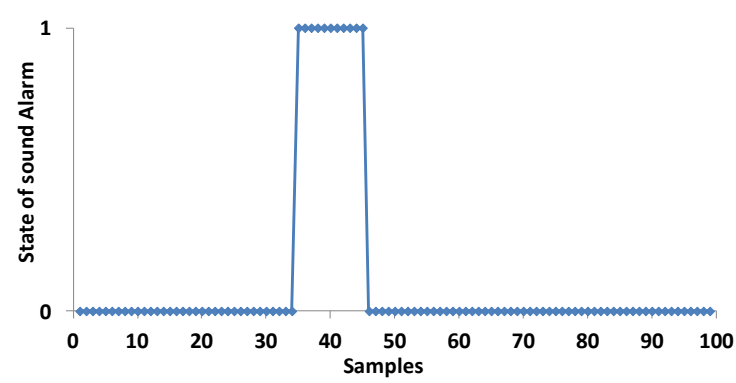

Figure 18. State of microphone alarm.
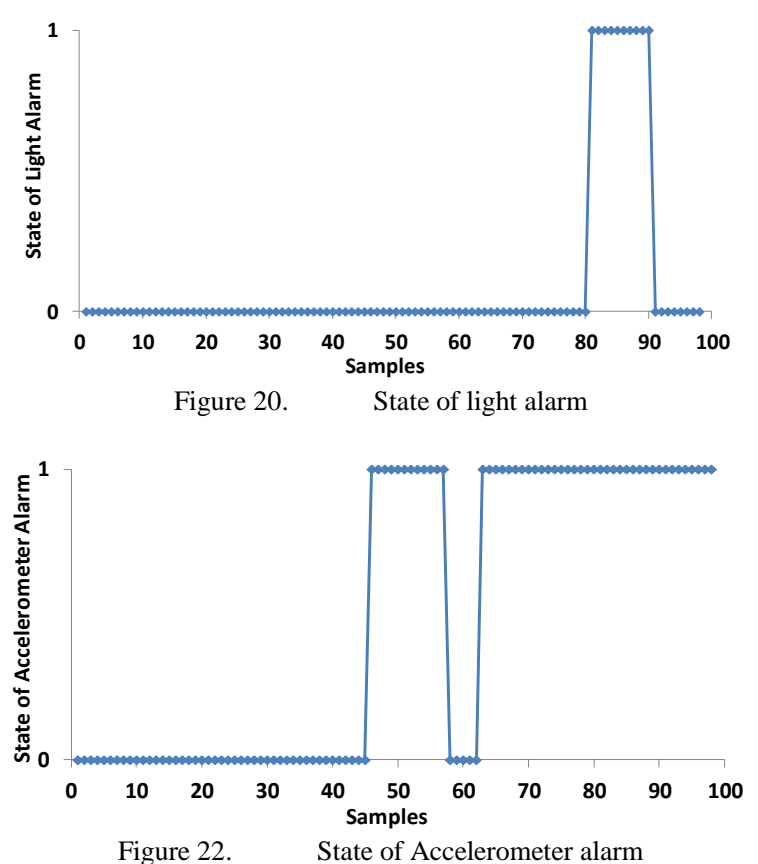

Following this approach, we have several cases where the generation of an alarm, from the information collected from a sensor, could induce to an error. This fact permits us to know several states that we can always consider as not dangerous states, so we define the initial utility value of the alarm action for these 
states as -1. Moreover, we can also define other states that can be always considered as dangerous, so we define the initial utility value of the alarm action for these states as 1 . For example, there could be cases where a person is playing and moving back to forth, thus it would activate the gyroscope alarm. However, if the person is close to the group, it would not be a reason to generate the alarm, but if it leaves the group, the alarm will be generated.

In this section we will see the behavior of several cases where the activation of a sensor could lead to false results, so they can be used to train the system. In Table 2 the decision rules derivate from these cases and their utility values for the alarm action are shown.

\begin{tabular}{|c|c|c|}
\hline Rules & $Q($ s, wait $)$ & $\begin{array}{c}Q(s, \text { Send } \\
\text { message alarm })\end{array}$ \\
\hline $\begin{array}{l}\text { State } s \text { with Gyroscope alarm }=1 \& \\
\text { GPS alarm }=0\end{array}$ & 1 & -1 \\
\hline $\begin{array}{l}\text { State } s \text { with Gyroscope alarm }=1 \& \\
\text { GPS alarm }=1\end{array}$ & -1 & 1 \\
\hline $\begin{array}{l}\text { State } s \text { with Accelorometer alarm }=1 \& \\
\text { GPS alarm }=0\end{array}$ & 1 & -1 \\
\hline $\begin{array}{l}\text { State } s \text { with Accelorometer alarm }=1 \& \\
\text { GPS alarm }=1\end{array}$ & -1 & 1 \\
\hline $\begin{array}{l}\text { State } s \text { with Compass alarm }=1 \& \\
\text { GPS alarm }=0\end{array}$ & 1 & -1 \\
\hline $\begin{array}{l}\text { State } s \text { with Compass alarm }=1 \& \\
\text { GPS alarm }=1\end{array}$ & -1 & 1 \\
\hline $\begin{array}{l}\text { State } s \text { with Sound alarm }=1 \& \\
\text { GPS alarm }=0\end{array}$ & 1 & -1 \\
\hline $\begin{array}{l}\text { State } s \text { with Sound alarm }=1 \& \\
\text { GPS alarm }=1\end{array}$ & -1 & 1 \\
\hline
\end{tabular}

Figure 23 shows the case of a person that is moving back to forth, but the alarm is not generated till the person leaves the group. Here we can see that before the $32^{\text {nd }}$ sample, nothing happens. But after this value the gyroscope alarm is activated, but no alarm message is sent. However, because after $46^{\text {th }}$ sample there is a significant increase of the distance of the affected person to the group, there will be a message alarm. Combining the response of both sensors, we obtain a combined alarm, which offers greater reliability than processing only the gyroscope sensor data.

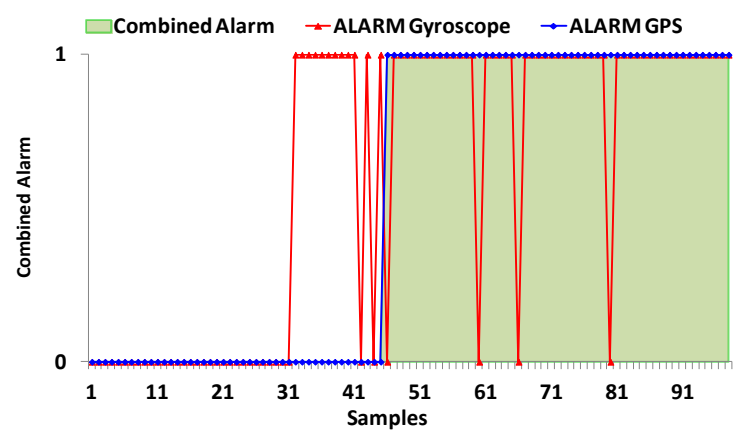

Figure 23. Combined alarm for GPS and Gyroscope sensors.

Another situation where a single sensor could generate a false positive, but it could be corrected by our smart collaborative protocol, is the following one. Imagine a new situation where there is a group of young people with some kind of deficiency. In this group, one of them starts to run around the group but without departing from the group. In this case, the acceleration sensor would generate an alarm. However, while the affected person is near the group, the situation is not necessarily a hazard. If GPS activates its alarm, jointly with the accelerometer alarm, there will be an emergency situation for the affected person, so the alarm message will be sent. Figure 24 shows the behavior of this case. We can see that although the accelerometer alarm has been activated, only when the distance from the affected person to the group increases exceeding the limit established as safe, the combined alarm of both sensors will send the alarm message. We may also note that although the accelerometer alarm is disabled, as the distance of the affected person to the group, is high, the combined alarm will persist. 


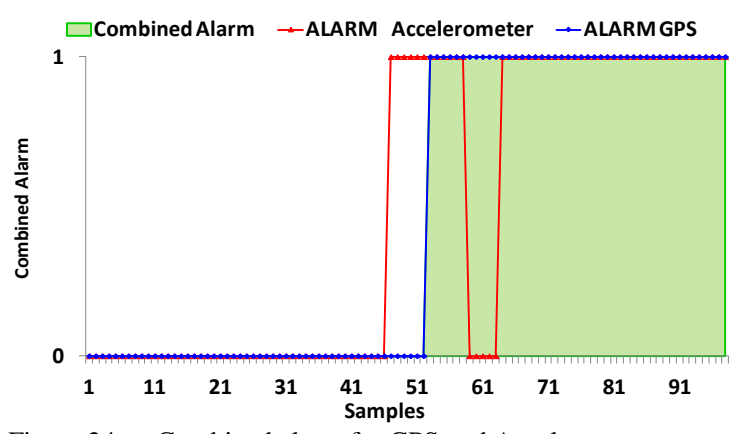

In the following situation, we have a person who begins to spin on its own, or even walk backwards, but always within the group. The compass sensor generates an alarm, because its value compared with their neighbors exceeds the specified threshold as normal, but no alarm message is generated until the person leaves the group. Figure 25 shows this situation where, although the compass sensor alarm is activated from the sample $25^{\text {th }}$, only when the GPS alarm is activated, the combined alarm is activated and thus the ala message is sent.

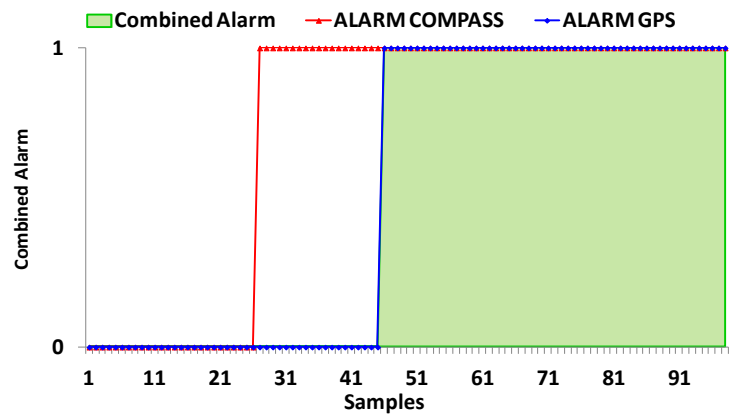

Figure 25. Combined alarm for compass and GPS sensors

Finally, we will show a case where there is an alarm generated by the noise. It is activated because it detects an increment of the noise level. But it does not necessarily mean a dangerous situation for the person who is screaming. However, if the sound alarm is activated while the distance of the affected person to the group is also increasing, it could mean that the person has suffered an accident and cannot follow the group. Figure 26 describes this situation. We can see that the combined alarm is only active when the distance to the group increases.

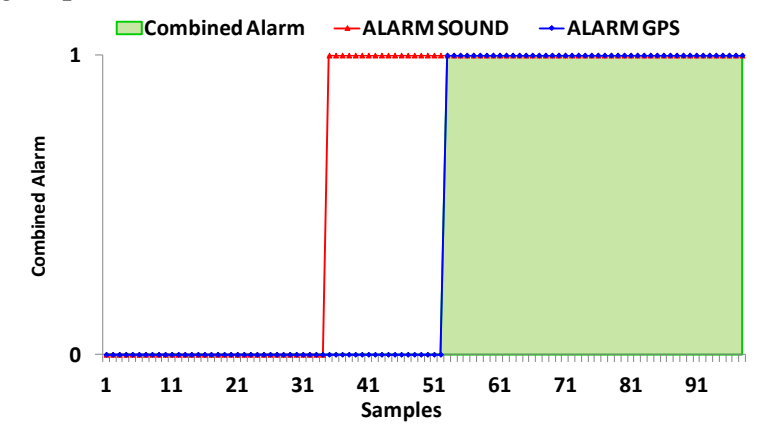

Figure 26. Combined alarm for GPS and microphone sensors.

\subsection{Ad hoc Network routing protocol}

In order to communicate all network devices, we should choose the most appropriate routing protocol for our network. In order to do this, we have simulated and compared 4 of the most used routing ad hoc protocols for a medium size group of persons. We have used the ns-2 simulator, which allows generating any kind of topology. Figure 27 shows the topology employed for our simulations. 


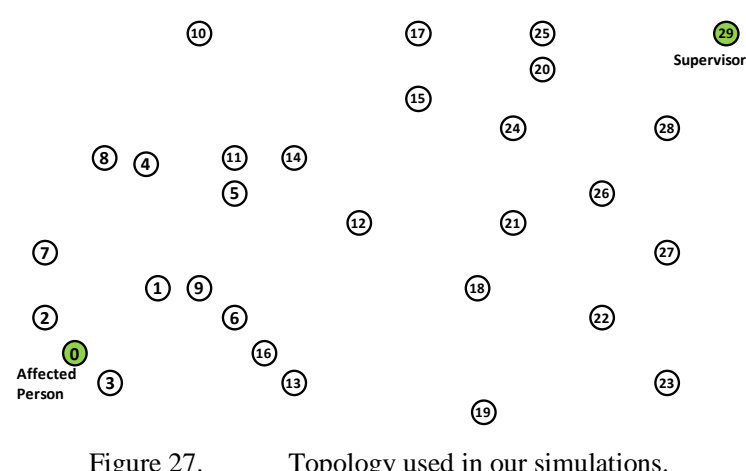

In figure 27 we can see that node " 0 " represents the person suffering the accident and node "29" is the supervisor. Protocols used in this comparison are:

- Ad hoc On-Demand Distance Vector (AODV).

- Destination-Sequenced Distance-Vector Routing (DSDV).

- Dynamic Source Routing (DSR).

- Optimized Link State Routing (OLSR).

After simulating these 4 protocols, we have obtained some parameters that are very useful for our study.

In Table 3 we present the comparison of the main parameters to evaluate routing protocols, such as: the time of reception of the first packet of the alarm message to the supervisor, the chosen route with the number of hops between nodes and the network overhead. We can see that the time needed to receive the first packet by the supervisor in proactive protocols vary between 10.19 seconds for OLSR and 72.30 seconds for DSDV, while for the reactive routing protocols, AODV and DSR, the first packet arrives at the supervisor position in less than a half of a second.

\begin{tabular}{c|cccc}
\hline Protocol & $\begin{array}{c}\text { Time of reception } \\
\text { of the first packet }\end{array}$ & Route & $\begin{array}{c}\text { Number of } \\
\text { hops }\end{array}$ & Overhead \\
\hline DSDV & $72.30 \mathrm{~s}$ & $0-9-14-15-20-28-29$ & 6 & $22 \%$ \\
AODV & $0.09 \mathrm{~s}$ & $0-9-5-12-15-24-28-29$ & 7 & $1 \%$ \\
DSR & $0.13 \mathrm{~s}$ & $0-9-14-15-24-28-29$ & 6 & $29 \%$ \\
OLSR & $10.19 \mathrm{~s}$ & $0-9-14-15-25-28-29$ & 6 & $67 \%$ \\
\hline \multicolumn{5}{c}{ Table 3 Comparison of the routing protocol's principal features }
\end{tabular}

Figure 28 shows the number of packets in the network as a function of the time, so we can clearly see the behavior of the network in proactive routing protocols when the routing tables converge. For OLSR this convergence occurs approximately in 10 seconds and for DSDV in approximately in 70 seconds.

As we can see, proactive routing protocols like DSDV and OLSR introduce higher number of packets in the network than the others because they have to keep the routing tables updated. DSDV has a mean value of 135.18 packets per second with a standard deviation of 64.66 packets per second, and OLSR presents an average of 399.16 packets per second and a standard deviation of 164.5 packets per second. On the other hand, reactive routing protocols, AODV and DSR, introduce a smaller amount of packets into the network compared with the proactive protocol. Concretely, in AODV protocol we observed a mean value of 157.37 packets per second with a standard deviation of 16.19 packets per second and for DSR an average of 147.57 and a standard deviation of 15.75 packets per second.

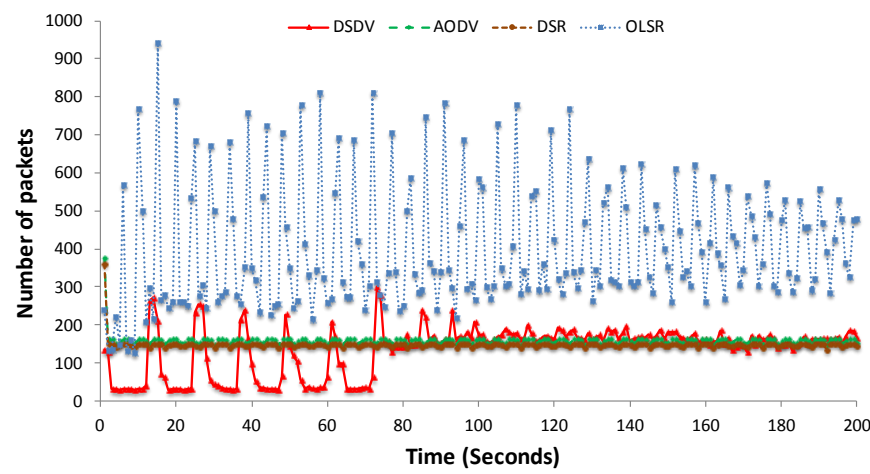


Figure 28. Volume of packets in the network, for the four protocols, in function of the time.

Figure 29 shows the amount of kb delivered through the network as a function of the time. As we know, OLSR routing protocol is one of most used ones in ad hoc networks, however, it presents the biggest number of transmitted bits. This is because OLSR is a proactive routing protocol, where all paths to all possible destinations are calculated a priori, keeping regular updates.

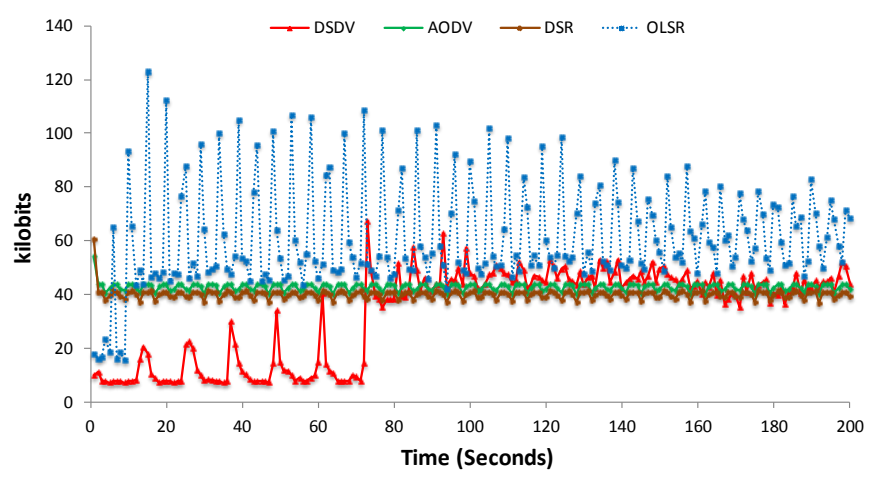

Figure 29. Volume of kilobits in the network, for the four protocols, in function of the time

The main disadvantage of this protocol is that it introduces overhead on the network, even on networks with few traffic flow. However, it can select valid routes almost immediately. In this case, we can estimate that OLSR shows a data flow close to $80 \mathrm{~kb}$, along the entire simulation time. Its average value of $\mathrm{kb}$ in the network is around $61 \mathrm{~kb}$. On the other hand, DSDV is a proactive routing protocol based on routing tables, where each network node maintains a routing table containing all possible destinations and the number of hops that a packet should travel to the destination. As we see, update messages must be sent continually through the network in order to maintain the consistency of the tables. However, the size of these packets is much lower than those used by OLSR. This makes to have an average amount of information in the network around $33 \mathrm{~kb}$. Lastly, we have AODV and DSR. They are reactive routing protocols, i.e., routes to find a path between senders and receivers are calculated only when they are needed to send information. For this reason, it has fewer network overheads. AODV has an average data volume of $42.7 \mathrm{~kb}$, while DSR presents an average value of $40 \mathrm{~kb}$.

\section{Conclusion}

Old and elderly people have intrinsic problems that should be monitored, especially when they are doing an outdoor activity. Falls in the elderly and disabled people can have very damaging results for them. Moreover, it is also very difficult to prevent falls in these people. In recent years, it has also appeared many degenerative diseases, which cause the disorientation of a person and may be lost. It is very complicated to control these situations. However, we can reduce the time to assist them by a supervisor or by the medical services.

It is very common to see a tour of a group of elderly and disabled people visiting a city. In these cases it is very difficult to control the persons inside the group.

In this paper, we present a smart collaborative algorithm for monitoring elderly and disabled people, which is able to collect sensor values from their mobile devices and compare them with those obtained by their neighbors, in order to determine whether it is producing an emergency situation or not. Furthermore, this algorithm is based on the reinforcement learning techniques, which will allow our system to learn about past situations. This feature allows the system to improve its accuracy and precision in the generation of alarms. Our system is able to predict an alarm before it occurs.

We have also studied that a single sensor could induce the generation of false positives, so in our system we use a combination of sensors, to avoid the false positives.

The protocols that provided better performance are DSR and DSDV. Each one is better than the others depending on the network features. If our system is monitoring elderly people with reduced mobility, we should use DSDV, since this protocol sends fewer packets to the network (and less kb) when the network has converged. However, if the network is being used by young people, with some kind of deficiency, the network will have greater mobility. In this case we should use the DSR protocol because this protocol 
consumes less bandwidth when there is mobility and after the network convergence. AODV only should be used for networks in very few devices, where the transmission time would be the most important factor. Finally OLSR is discarded for our network because it does not provide good performance in our case. These simulations allowed us to check the performance of our system when some of the main ad hoc routing protocols are running and see which ones perform better according to some specific cases.

Given the benefits of adding location information in our messages [31], in a future work we will include a positioning method to our system (for both, indoors [32] [33] and outdoors [34] [35]) in order to provide not only the alarm but also where the disable or elderly person is placed. Moreover, we will include advanced smartphone storage systems [36] in order to save locally the sensed data for future studies or to include a self-learning system in our system.

\section{Acknowledgements}

This work has been partially supported by the Instituto de Telecomunicações, Next Generation Networks and Applications Group (NetGNA), Portugal, and by National Funding from the FCT Fundação para a Ciência e a Tecnologia through the PEst-OE/EEI/LA0008/2011 Project.

\section{References}

[1] Cisco Systems Inc. "Cisco Visual Networking Index: Global Mobile Data Traffic Forecast Update, 2010-2015," White Paper, February 1, 2011.

[2] O. Pereira, J. Caldeira, J. Rodrigues, Body Sensor Network Mobile Solutions for Biofeedback Monitoring, Journal of Mobile Networks and Applications, 2011, Vol. 16, Issue 6, pp. 713-732.

[3] Google. Galaxy nexus (2012). Available: http://www.google.com/nexus/.

[4] E. Commission, "Demography report 2010," Eurostat, the Statistical Office of the European Union, 2010. At http://ec.europa.eu/social/BlobServlet?docId=6824\&langId=en

[5] K.E. Thomas, J.A. Stevens, K. Sarmiento, M.M. Wald, Fall-related traumatic brain injury deaths and hospitalizations among older adults-United States, 2005, Journal of Safety Research, 2008, vol. 39, Issue 3, pp. $269-272$.

[6] G. Fortino, R. Giannantonio, R. Gravina, P. Kuryloski, R. Jafari, "Enabling Effective Programming and Flexible Management of Efficient Body Sensor Network Applications", IEEE Transactions on Human-Machine Systems, vol. 43, no. 1, pp. 115-133, Jan. 2013. DOI: 10.1109/TSMCC.2012.2215852.

[7] F. Bellifemine, G. Fortino, R. Giannantonio, R. Gravina, A. Guerrieri, M. Sgroi, "SPINE: A domain-specific framework for rapid prototyping of WBSN applications" Software Practice and Experience, 41(3), 2011, pp. 237-265,

[8] Elsa Macias, Jaime Lloret, Alvaro Suarez and Miguel Garcia, Architecture and Protocol of a Semantic System Designed for Video Tagging with Sensor Data in Mobile Devices, Sensors, (Feb. 2012) Vol. 12, Issue 2. Pp. 2062-2087.

[9] S. Sendra, E. Granell, J. Lloret, J. J. P. C. Rodrigues, Smart Collaborative System Using the Sensors of Mobile Devices for Monitoring Disabled and Elderly People, 3rd IEEE International Workshop on Smart Communications in Network Technologies, Ottawa, Canada, June 11, 2012.

[10] N. Lane, E. Miluzzo, H. Lu, D. Peebles, T. Choudhury, A. Campbell, A survey of mobile phone sensing, IEEE Communications Magazine, 2010, Vol. 48, Issue 9, pp. 140-150.

[11] C. Muldoon, G. OHare, M. OGrady, Collaborative agent tuning: Performance enhancement on mobile devices Engineering Societies in the Agents World VI, Lecture Notes in Computer Science, Volume 3963/2006, 2006, pp. 241-258.

[12] H. Turner, J. White, C. Thompson, K. Zienkiewicz, S. Campbell, D.C. Schmidt, Building Mobile Sensor Networks Using Smartphones and Web Services: Ramifications and Development Challenges, Handbook of Research on Mobility and Computing, Hershey, PA. ,2009. Available: http://lsrg.cs.wustl.edu/ schmidt/PDF/new-ww-mobile-computing.pdf.

[13] A. Kansal, M. Goraczko, F. Zhao, Building a sensor network of mobile phones, 6th International Conference on Information Processing in Sensor Networks. Cambridge, Massachusetts, USA, April 24 - 27, 2007 pp. 547-548.

[14] I. Plaza, L. Martín, S. Martin, C. Medrano, Mobile applications in an aging society: Status and trends, Journal of Systems and Software, 2011, Vol 84, Issue 11, pp 1977-1988.

[15] L. Camarinha-Matos, H. Afsarmanesh, Telecare: Collaborative virtual elderly support communities, 1st Workshop on TeleCare and Collaborative Virtual Communities in Elderly Care, Porto, Portugal, 13 April, 2004.

[16] B. Chen, D. Pompili, Transmission of Patient Vital Signs Using Wireless Body Area Networks, Journal of Mobile Networks and Applications, 2011, Vol. 16, Issue 6, pp. 663-682.

[17] J. Dai, X. Bai, Z. Yang, Z. Shen, D. Xuan, "Mobile phone-based pervasive fall detection," Personal and ubiquitous computing, 2010, Vol. 14, Issue 7, pp. 633-643.

[18] P. Martin, M. A. Sánchez, L. Álvarez, V. Alonso, J. Bajo, Multiagent system for detecting elderly people falls through mobile devices, International Symposium on Ambient Intelligence (ISAmI'11), Salamanca (Spain) 6-8 April 2011

[19] P.N. Fahmi, V. Viet, C. Deok-Jai, "Semi-supervised fall detection algorithm using fall indicators in smartphone," Proceedings of the 6th International Conference on Ubiquitous Information Management and Communication, 2012, pp. 122.

[20] M. Sánchez, P. Martín, L. Álvarez, V. Alonso, C. Zato, A. Pedrero, J. Bajo, A New Adaptive Algorithm for Detecting Falls through Mobile Devices, Trends in Practical Applications of Agents and Multiagent Systems, 2011, pp. 17--24. 
[21] M. Fahim, I. Fatima, S. Lee, and Y.K. Lee, Daily Life Activity Tracking Application for Smart Homes using Android Smartphone, 14th International Conference on Advanced Communication Technology, Yongin, South Korea, 19-22 February 2012 , pp. $241-245$.

[22] B. Kaluža, V. Mirchevska, E. Dovgan, M. Luštrek, M. Gams, An agent-based approach to care in independent living, Ambient Intelligence, Lecture Notes in Computer Science, 2010, vol. 6439, pp. 177-186.

[23] A. Costa, G. Barbosa, T. Melo, P. Novais, Using mobile systems to monitor an ambulatory patient, in International Symposium on Distributed Computing and Artificial Intelligence, Advances in Intelligent and Soft Computing, 2011, vol. 91, pp. 337-344.

[24] R. Olfati-Saber, J. Fax, R. Murray, Consensus and cooperation in networked multi-agent systems, Proceedings of the IEEE, 2007, vol. 95, Issue 1, pp. 215-233.

[25] A. Arcelus, M. H. Jones, R. Goubran, F. Knoefel, Integration of smart home technologies in a health monitoring system for the elderly, 21st International Conference on Advanced Information Networking and Applications Workshops, 2007, Vol. 2, pp. $820--825$.

[26] H. Kahmen, W. Faig, Surveying, NewYork: Walter de Gruyter \& Co., Berlin (Germany) 1988.

[27] Sol LM870 mobile phone features. Available at: http://es.made-in-china.com/co_runrise/product_Dual-SIM-Card-DualStandby-GPS-Temperature-UV-Sensor-Pedometer-Sunrise-LM870-Mobile-Phone_hesighyiy.html.

[28] STLM20 temperature sensor features. Datashet available http://www.st.com/internet/com/TECHNICAL_RESOURCES/TECHNICAL_LITERATURE/DATASHEET/CD00119601.pdf.

[29] S. Sendra, J. Lloret, M. Garcia and J. F. Toledo, Power saving and energy optimization techniques for Wireless Sensor Networks, Journal of Communications, Vol. 6 Issue 6, Pp. 439-459. August 2011.

[30] Matlab Website. Available at: www.mathworks.com/products/matlab.

[31] A. Pal, Localization Algorithms in Wireless Sensor Networks: Current Approaches and Future Challenges, Network Protocols and Algorithms, 2010, Vol 2, Issue 1, Pp. 45-74.

[32] M. Garcia, F. Boronat, J. Tomás, J. Lloret, The Development of Two Systems for Indoor Wireless Sensors Self-location, Ad Hoc \& Sensor Wireless Networks, Vol. 8, Issue 3-4, Pp. 235-258. 2009,

[33] J. Lloret, J. Tomás, M. Garcia, A. Cánovas, A Hybrid Stochastic Approach for Self-Location of Wireless Sensors in Indoor Environments, Sensors, 2009, Vol. 9, Issue 5, Pp. 3695-3712.

[34] M. Garcia, S. Sendra, C. Turro, J. Lloret, User's Macro and Micro-mobility Study using WLANs in a University Campus, International Journal On Advances in Internet Technology, 2011, Vol. 4, Issue 1\&2, Pp. 37-46.

[35] J. Lloret, J. Tomas, A. Canovas, I. Bellver, GeoWiFi: A Geopositioning System Based on WiFi Networks, The Seventh International Conference on Networking and Services (ICNS 2011), Venice (Italy), May 6-10, 2011.

[36] W. Yu, X. Su, J. Hansen, A Smartphone Design Approach to User Communication Interface for Administering Storage System Network, Network Protocols an Algorithms. Vol 4, No 4 (2012). Pp. 126-155 